\title{
Immunohistochemical detection of human papillomavirus capsid proteins L1 and L2 in squamous intraepithelial lesions: potential utility in diagnosis and management
}

\author{
Anna Yemelyanova ${ }^{1}$, Patti E Gravitt ${ }^{2,3,4}$, Brigitte M Ronnett ${ }^{1,5}$, Ann F Rositch ${ }^{3,4}$, \\ Aleksandra Ogurtsova ${ }^{1}$, Jeffrey Seidman ${ }^{6}$ and Richard BS Roden ${ }^{1,5}$ \\ ${ }^{1}$ Department of Pathology, Johns Hopkins University School of Medicine, Baltimore, MD, USA; \\ ${ }^{2}$ Perdana University Graduate School of Medicine, Serdang, Malaysia; ${ }^{3}$ Department of Epidemiology, \\ Johns Hopkins University Bloomberg School of Public Health, Baltimore, MD, USA; ${ }^{4}$ Department of \\ Molecular Microbiology and Immunology, Johns Hopkins University Bloomberg School of Public Health, \\ Baltimore, MD, USA; ${ }^{5}$ Department of Gynecology and Obstetrics, Johns Hopkins University School of \\ Medicine, Baltimore, MD, USA and ${ }^{6}$ Department of Pathology, Washington Hospital Center, Washington, \\ $D C, U S A$
}

\begin{abstract}
While cervical cancer screening relies on cervical cytology and high-risk human papillomavirus (HPV) detection, the histologic diagnosis, and specifically lesion grade, is the main parameter that drives clinical management of screen-positive women. Morphologically diagnosed squamous intraepithelial lesions (SIL/CIN) regress spontaneously in more than half of the cases, but identifying those likely to persist and progress is not currently possible based upon morphology. Lack of major capsid protein L1 expression has been suggested as a feature in progressive lesions, whereas expression of the minor capsid protein L2 has not been extensively evaluated. The goal of this study is to evaluate immunohistochemical expression of L1 and L2 in SILs in correlation with lesion grade. A total of 150 cervical specimens with SILs were selected based on HPV 16 or HPV 18 detection by Q-PCR. These included 89 low-grade SILs (LSIL/CIN 1) and 123 high-grade SILs (75 HSIL/CIN 2 and 48 HSIL/CIN 3). More than one lesion/grade was identified in 53 specimens. The presence and grade of SIL was determined by a panel of pathologists. Capsid protein expression was assessed by immunohistochemistry using MAB 837 for L1 and RG-1 for L2. Lesions of different grades in the same specimen were scored separately. Expression of capsid proteins was detected in 34/89 (40\%) LSIL/CIN 1, 5/75 (6\%) HSIL/CIN 2 and none of 48 HSIL/CIN 3 . L1 and L2 were co-expressed in the same area of the lesion in 22 cases. In addition, L1 alone was expressed in 6 lesions and L2 alone in 11 lesions. Among the cases with multiple lesion grades in the same specimen, none with HSIL/CIN 3 expressed capsid proteins in any portion/grade of the lesion. HPV capsid proteins are expressed almost exclusively in LSIL/CIN 1 and rarely in HSIL/CIN 2. Additional studies are warranted to examine lack of L1 and L2 expression in LSIL/CIN 1 as a predictor of persistence or progression to HSIL/CIN 3, the precursor of cervical cancer.

Modern Pathology (2013) 26, 268-274; doi:10.1038/modpathol.2012.156; published online 21 September 2012
\end{abstract}

Keywords: biomarkers; capsid proteins; cervical cancer precursors; HPV; immunohistochemistry

Although the incidence of invasive cervical cancer in the United States is decreasing, the rates of viral

Correspondence: Dr A Yemelyanova, MD, Department of Pathology, Johns Hopkins University School of Medicine, 401 North Broadway, Weinberg Building, Room 2242, Baltimore, MD 21231, USA.

E-mail: ayemely1@jhmi.edu

Received 22 May 2012; revised 19 July 2012; accepted 19 July 2012; published online 21 September 2012 infection leading to development of carcinoma precursors (squamous intraepithelial lesions (SILs)) are extremely high. Management of SILs remains an important health-care problem despite recent advances, including the development of effective prophylactic human papillomavirus (HPV) vaccines. Although cervical cancer screening relies on cervical cytology and high-risk HPV detection, the histologic diagnosis, and specifically lesion grade, is the main parameter that drives clinical management 
of screen-positive women. Histologically diagnosed SILs (traditionally labeled as cervical intraepithelial neoplasia (CIN)) regress spontaneously in more than half of the cases, but morphology alone is an insufficient predictor of lesion behavior. ${ }^{1-3}$ Development of markers that can predict the lesion regression versus persistence/progression would be extremely useful for clinical management.

It has been suggested that the expression and distribution of viral gene products can serve as potential markers for distinction of regressive versus progressive lesions. ${ }^{4-6}$ These studies indicate that the inability of virus to complete its productive cycle is associated with increased lesion grade.

Productive viral infection is characterized by genome amplification and expression of late viral genes responsible for virion assembly, notably the capsid proteins (L1 and L2). This late gene expression is restricted to terminally differentiated, superficial squamous epithelial cells. Major capsid protein L1 constitutes the primary structural element of viral capsid. It also represents a major target for the cell-mediated immune response. L2 is a minor component of the capsid, thought to aid in the assembly and the packaging of viral DNA within the virions. ${ }^{7}$ Aberrant squamous cell differentiation that is considered a feature of a high-grade SIL is associated with a failure to express the capsid proteins and thus to complete the life cycle. Absence of L1 expression was a feature of progressive lesions in several studies using cytologic specimens. ${ }^{8,9}$ Expression of L2 protein in clinical lesions has not been extensively evaluated, although it has been suggested that L2 is also expressed predominantly in LSIL/CIN 1, consistent with productive infection in these low-grade lesions. ${ }^{10}$ The goal of this study is to evaluate immunohistochemical expression of L1 and L2 in SILs in correlation with lesion grade.

\section{Materials and methods}

\section{Case Selection}

The study was approved by the Johns Hopkins University School of Medicine Institutional Review Board. A total of 342 cervical specimens including cervical biopsies and excisional specimens (loop electrosurgical excision procedure (LEEP) and cone biopsy specimens) were retrieved from the files of the Johns Hopkins Hospital. Of these, 150 cervical tissue specimens containing 212 morphologically distinct SILs were selected for analysis based on HPV 16 or HPV 18 DNA detection by Q-PCR (see below). The study was limited to HPV 16- and HPV 18-positive cases to match the specificity of the antibodies used for immunohistochemical analysis of L1 and L2 expression. The 212 SILs included 89 low-grade SILs (LSIL/CIN 1) and 123 high-grade SILs (HSILs), with the latter including 75 CIN 2 and
48 CIN 3 (53 cases had more than one grade of lesion in the same specimen). Of these 150 samples, 112 contained HPV 16 DNA and 32 contained HPV 18 DNA; both HPV types were detected in 6 specimens (adjacent SILs of different grades present in the same specimen were not separately analyzed for HPV type). The presence and the grade of SILs were confirmed by thorough histologic review by two observers (AY and BMR).

\section{HPV DNA Detection}

DNA was isolated from two $10-\mu \mathrm{m}$ paraffin-embedded tissue sections after octane deparaffinization and digestion with buffer containing proteinase $\mathrm{K}$. A $2-5 \mu \mathrm{l}$ aliquot of purified DNA was tested for the presence and number of copies of HPV 16 DNA and HPV 18 DNA as previously described using realtime TaqMan PCR methods. ${ }^{11}$ All viral quantities were normalized to total human cell equivalents tested by quantitating the ERV-3 human endogenous retrovirus gene from each sample. ${ }^{12}$

\section{Immunohistochemical Analysis}

For immunohistochemical analysis, $4-5 \mu \mathrm{m}$ thick tissue sections were deparaffinized in xylene and rehydrated. Epitope retrieval was performed by placing the slides in Citrate Buffer Solution $(\mathrm{pH}$ 6.0, ZYMED Laboratories, Life Technologies, Carlsbad, CA, USA) at $95^{\circ} \mathrm{C}$ for $20 \mathrm{~min}$. Endogenous peroxidase activity was blocked with Peroxidase Blocking Solution (DAKO North America, Carpinteria, CA, USA), and then the slides were incubated with Protein Block (Biogenex, Fremont, CA, USA) for $30 \mathrm{~min}$. Primary antibody incubation was performed for $1 \mathrm{~h}$ at room temperature with MAB 837 clone 1H8 (1:1000 dilution; final antibody concentration $1 \mu \mathrm{g} / \mathrm{ml}$; Chemicon/Millipore, Billerica, MA, USA) for L1 and for 1.5 h with RG-1 (1:500; final antibody concentration $3.1 \mu \mathrm{g} / \mathrm{ml}$ ) for L2. ${ }^{13}$ These antibodies recognize the respective capsid proteins of HPV 16 and 18; MAB 837 also recognizes HPV 1, 6, 11 and 31; the specificity of RG-1 for other HPV types is less well characterized. Staining was detected with anti-mouse HRP Polymer (DAKO) for $30 \mathrm{~min}$ at room temperature. Staining was repeated in triplicate in all positive cases, all cases with discordant L1/L2 expression and in a random $10 \%$ of negative cases; all results were consistently reproduced. In cases with more than one grade of SIL present in the same slide, immunostaining in each distinct grade was analyzed separately. Any nuclear staining was considered a positive result, regardless of the quantity of cells having expression. For descriptive purposes, the extent of the staining was also semiquantitatively assessed: positive, $\geq 10$ positive nuclei; focal positive, $>2$ but $<10$ positive nuclei; or limited positive, 1 or 2 positive nuclei. Expression of either L1 or L2 or both was considered 
as evidence of capsid protein expression for statistical analysis. In the cases with multiple lesions/grades present, the expression was recorded for each grade separately and reported as such. For the purposes of data presentation, the following categories were distinguished: LSIL alone-the only grade present in the specimen; LSIL with HSIL/CIN 2-coexisting LSIL and HSIL/CIN 2 within the same specimen, but the expression is assessed in LSIL only; LSIL with HSIL/CIN 3-coexisting LSIL and HSIL/CIN 3 within the same specimen, but the expression is assessed in LSIL only; LSIL total-all LSILs alone or associated with a higher grade SIL. Similar categories were distinguished for HSIL/CIN 2 and HSIL/CIN 3 (HSIL/CIN 2 alone; HSIL/CIN 2 with LSIL; etc).

\section{Statistical Analysis}

The number and percentage of SIL cases expressing any L1 and/or L2 capsid protein are presented by each grade of lesion present in the specimen. If more than one morphologically distinct SIL was present in a specimen, the data are further stratified by co-occurring lesion grades. The $\chi^{2}$ test or Fisher's exact test statistics, when the number in any category was $\leq 5$, were calculated to compare the proportion of cases expressing any capsid protein between two mutually exclusive groups based on SIL grade: LSIL/CIN 1, HSIL/CIN 2 and HSIL/CIN 3. Cochran-Armitage test was used to determine if there was a significant trend in the proportion of cases expressing capsid protein across the ordered categories LSIL/CIN 1 alone, HSIL/CIN 2 alone and HSIL/CIN 3 alone. The level of significance was $P<0.05$ and no results were corrected for multiple comparisons. All analyses were conducted using SAS version 9.2 (SAS, Cary, NC, USA).

\section{Results}

Expression of either L1 or L2 or both was observed in 39 out of 212 lesions (18\%) overall. These included 34 LSIL/CIN 1 and 5 HSIL/CIN 2. Coexpression of both L1 and L2 in the same area of the lesion was observed in 22 lesions, including 18 LSIL/CIN 1 and 4 HSIL/CIN 2 (Figure 1). L1 alone was expressed in 6 lesions, all of which were LSIL/ CIN 1; L2 alone was expressed in 11 lesions, including 10 LSIL/CIN 1 and 1 HSIL/CIN 2. Neither L1 nor L2 was expressed in any of the 48 HSIL/CIN 3 lesions (Figure 2). The positive nuclei were always situated in the upper epithelial layers of the lesional squamous epithelium (Figure 1). The extent of staining was positive ( $\geq 10$ cells) in 21 lesions, focal positive in 13 lesions and limited positive in 5 lesions (Figure 1). The latter included 2 LSIL/CIN 1 and 3 HSIL/CIN 2. The immunohistochemical staining results in SILs stratified by grade are summarized in Table 1.
The difference in capsid protein expression in LSIL/CIN 1 alone (49\%) versus HSIL/CIN 2 alone $(13 \%)$ or any HSIL (CIN 2 and CIN 3; 5\%) was statistically significant $(P=0.0039$ and $P<0.0001$, respectively). There were no differences in expression between LSIL/CIN 1 alone $(49 \%)$ and LSIL/CIN 1 with HSIL/CIN $2(34 \%)$ groups $(P=0.2)$. However, expression in LSIL/CIN 1 with or without HSIL/CIN 2 (43\%) was different from expression in LSIL/CIN 1 with adjacent HSIL/CIN $3 \quad(0 \%)$ $(P=0.006)$. Within the HSIL/CIN 2 group, there were no differences in expression between HSIL/ CIN 2 alone (13\%) and HSIL/CIN 2 with LSIL/CIN 1 $(7 \%)$ groups $(P=0.108)$. However, expression in HSIL/CIN 2 with or without LSIL/CIN 1 (9\%) was different from expression in HSIL/CIN 2 with adjacent HSIL/CIN $3(0 \% ; P=0.008)$. The expression of capsid proteins decreased with increase in lesion grade (LSIL/CIN 1 alone vs HSIL/CIN 2 alone vs HSIL/CIN 3 alone; $\left.P_{\text {trend }}<0.0001\right)$.

\section{Discussion}

The need for an immunohistochemical marker of HPV-related precancer that is likely to progress is indisputable. The majority of the biomarkers used in pathologic diagnosis of SILs, including p16, PCNA, MCM and ProEx C, reflect the presence of a high-risk HPV-related lesion, but are not specific for identification of progressive disease with potential to develop into an invasive carcinoma. It has been suggested that patterns of viral protein expression may be used to differentiate between self-limited productive viral infection and a true precancer. ${ }^{6}$

Immunohistochemical detection of viral protein expression has been described in prior studies in cervical specimens. ${ }^{14-16}$ Kurman et $a l^{16}$ reported immunohistochemical expression of PV common (structural) proteins in $43 \%$ of mild dysplasia, 15\% of moderate dysplasia and only in a rare case of severe dysplasia. In one study, immunohistochemical expression of L1 and L2 in two cases of condyloma and a CIN 2 lesion was identified in nuclei restricted to the upper epithelial layers. ${ }^{14}$

A number of more recent studies have evaluated the expression of major capsid protein L1 in cytologic specimens. It has been shown that 30 $75 \%$ of LSILs and $33-40 \%$ of HSILs express L1 in cytology specimens. ${ }^{8,9,17-21}$ Some of these studies evaluated the prognostic utility of L1 immunocytochemistry on a Pap sample in predicting lesion behavior. Most of the studies that relied on 'cytologic regression' with variable length of follow-up (up to 2 years) associated the presence of L1 expression with clinical 'regression'. However, lesion regression is difficult to reliably establish in the clinical setting because histologic evaluation of the entire cervix is not possible in most cases and all other measures of outcome (Pap cytology or colposcopy with cervical biopsies) have imperfect 

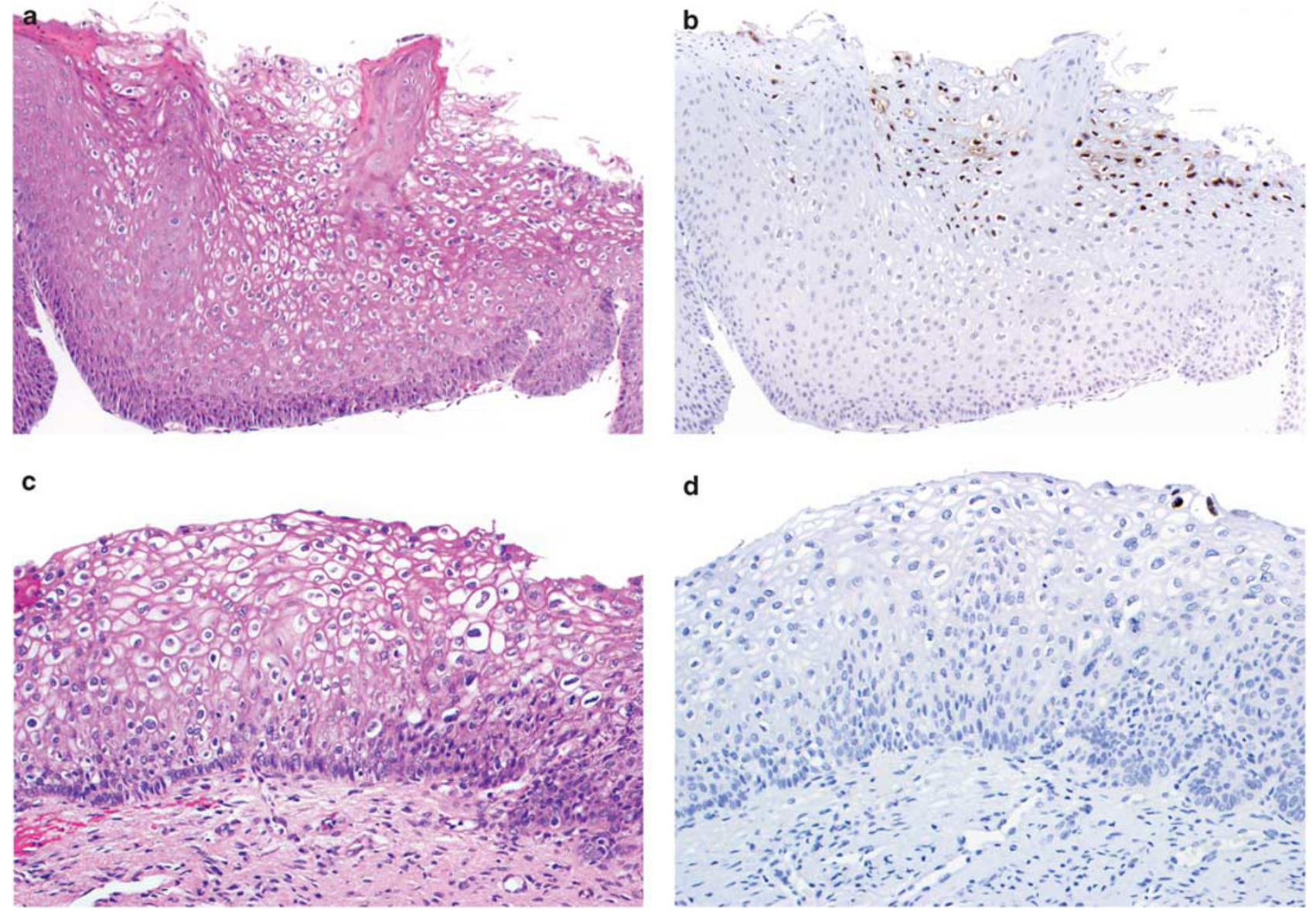

Figure 1 (a, c) Low-grade squamous intraepithelial lesion (LSIL/CIN 1). (b) Extensive L2 expression in the superficial epithelial layers; L1 showed similar findings (not shown). (d) Limited L1 expression (two positive nuclei) in the most superficial epithelial layer; the distribution of L2 expression was identical (not shown).

sensitivity for detecting significant lesions. In addition, the diagnostic biopsy itself in some cases may be curative and result in removal of the entire lesion.

Our data are consistent with previously published studies using histologic specimens that demonstrated L1 expression in $30-65 \%$ of LSIL/CIN $1 .{ }^{22-25}$ A lower proportion of the positive cases in histology compared with cytology specimens is likely related to the fact that in the majority of lesions the expression of capsid proteins is restricted to the most superficial layers of the epithelium. Thus, the keratinocytes in the superficial layers are most readily detached and harvested during sampling for a Pap test, but might also be inadvertently removed from the epithelial surface during colposcopy (mucous clearing etc.) and tissue handling during pathologic processing. A larger number of positive cases in cytology specimens compared with matched histologic preparations was previously described. ${ }^{8,26,27}$ Some studies evaluating the correlation of L1 and p16 expression in LSIL/CIN 1 have observed that lesions lacking L1 and demonstrating diffuse p16 expression are more likely to persist and progress. ${ }^{25}$ Diffuse p16 expression in these cases is most likely related to the presence of high-risk HPV within these lesions, most commonly HPV 16 and HPV 18; these types are associated with lower regression rates compared with LSIL/CIN 1 harboring other HPV types; ${ }^{28}$ thus, the prognostic value of p16 expression is likely not independent of the HPV type implicated in the lesion development.

In our study, a significant fraction of LSIL/CIN 1 cases were L1 negative, but the value of L1 expression alone as a predictor of behavior in tissue specimens is unclear. The inability to detect L1 in these LSIL/CIN 1 cases may reflect inadequate sampling, given the extremely focal staining in some cases noted in this and other studies. ${ }^{22}$ Using cytology Pap samples likely provides a more representative sample of superficial lesional cells for analysis of L1 expression.

We have previously reported expression of L2 in 89\% of LSIL/CIN 1 lesions and none of HSIL/CIN 2 and HSIL/CIN 3 in a study with a smaller number of cases. ${ }^{10}$ These data and our current data are different from what has been previously reported in another prior study that observed L2 expression in a significant proportion of moderate and severe 

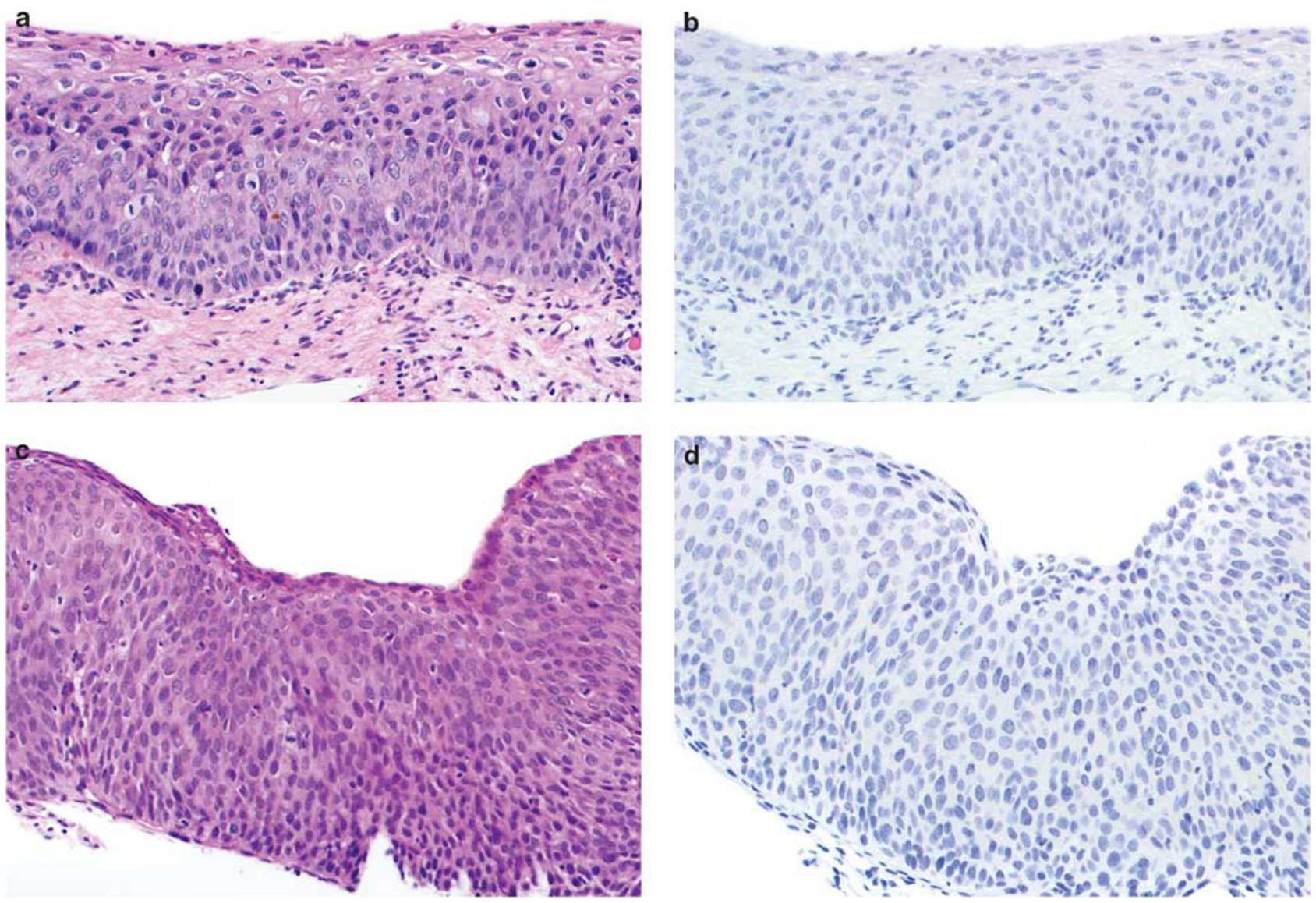

Figure 2 (a) High-grade squamous intraepithelial lesion (HSIL/CIN 2). (b) Absent expression of L1; L2 showed similar findings (not shown). (c) High-grade squamous intraepithelial lesion (HSIL/CIN 3). (d) The lesion lacks expression of L1; L2 expression was absent as well (not shown).

dysplasias; ${ }^{29}$ this could be because of differences in histologic grading.

The discordant expression of L1 and L2 in the same lesion that was observed in 17 cases $(43 \%$ of positive cases) in our study is surprising. This may be related to infection with multiple HPV types, with discordant expression related to antibody specificity in the setting of an HPV type other than HPV 16 or HPV 18. Thus, detection of both L1 and L2 proteins might not have added value if a more broadly HPV type-specific antibody for either L1 or L2 had been used.

All cases of HSIL/CIN 3, either alone or with adjacent LSIL/CIN 1 or HSIL/CIN 2, lacked expression of capsid proteins. Statistically, expression of capsid proteins was negatively associated with the grade of the lesion $\left(P_{\text {trend }}<0.0001\right)$.

The absence of capsid protein expression observed in this study differs from data reported by Galgano et $\mathrm{al}^{30}$ that recorded L1 expression in $16.5 \%$ of HSIL/CIN 3. These discordant findings could be related to fact that the current study was restricted to HPV 16 and HPV 18-related lesions, whereas the reported expression of L1 in HSIL/CIN 3 in the study of Galgano et $a l^{30}$ was based on SILs irrespective of HPV type. Reported L1 staining in HSIL/CIN 3 in some other studies may also be related to the known worldwide difference in histologic grading of SILs.

Recent studies evaluating methylation of HPV genes, specifically L1, found high levels of methylation of this gene associated with high-grade cytology. ${ }^{31,32}$ Consistent intense methylation of L1 gene was shown in HPV 18-related cervical carcinomas. ${ }^{33}$ These findings may provide further insight into the mechanisms of loss of capsid protein expression in higher-grade SILs.

In our study, $62 \%$ of all LSIL/CIN 1 lacked expression of both L1 and L2. The significance of this finding is not clear. Lack of detected expression in some of the negative cases could be a reflection of focality of staining and sampling. In other cases, it could represent a false-negative result related to the presence of HPV types other than those that are covered by the antibodies used. Nonetheless, further investigation of the viral capsid protein expression as a marker of progression/persistence in LSILs in correlation with methylation analysis of the viral genome is warranted.

Eleven specimens contained discrete foci of both LSIL/CIN 1 and HSIL/CIN 3 (with or without HSIL/ CIN 2). No expression of either L1 or L2 was observed in HSIL/CIN 3 foci. Interestingly, in these cases with 
Table 1 Expression of HPV capsid proteins (L1 and L2) in squamous intraepithelial lesions

\begin{tabular}{|c|c|c|}
\hline $\begin{array}{l}\text { Squamous } \\
\text { intraepithelial } \\
\text { lesion grade }\end{array}$ & $\begin{array}{l}\text { Number of } \\
\text { lesions/grade } \\
\text { (n) }\end{array}$ & $\begin{array}{c}\text { Number of lesions } \\
\text { expressing capsid proteins, } \\
\mathrm{n}(\%)^{\mathrm{a}}\end{array}$ \\
\hline LSIL/CIN 1-total & 89 & $34(38 \%)$ \\
\hline LSIL/CIN 1 alone & 49 & $24(49 \%)$ \\
\hline $\begin{array}{l}\text { LSIL/CIN } 1 \text { with } \\
\text { HSIL/CIN } 2\end{array}$ & 29 & $10(34 \%)$ \\
\hline $\begin{array}{l}\text { LSIL/CIN } 1 \text { with } \\
\text { HSIL/CIN } 3\end{array}$ & 2 & $0(0 \%)$ \\
\hline $\begin{array}{l}\text { LSIL/CIN } 1 \text { with } \\
\text { HSIL/CIN } 2 \text { and } \\
\text { HSIL/CIN } 3\end{array}$ & 9 & $0(0 \%)$ \\
\hline $\begin{array}{l}\text { HSIL CIN } 2 \text { and } \\
\text { CIN 3-total }\end{array}$ & 123 & $5(4 \%)$ \\
\hline HSIL/CIN 2-total & 75 & $5(7 \%)$ \\
\hline HSIL/CIN 2 alone & 24 & $3(13 \%)$ \\
\hline $\begin{array}{l}\text { HSIL/CIN } 2 \text { with } \\
\text { LSIL/CIN } 1\end{array}$ & 29 & $2(7 \%)$ \\
\hline $\begin{array}{l}\text { HSIL/CIN } 2 \text { with } \\
\text { HSIL/CIN } 3\end{array}$ & 13 & $0(0 \%)$ \\
\hline $\begin{array}{l}\text { HSIL/CIN } 2 \text { with } \\
\text { LSIL/CIN } 1 \text { and } \\
\text { HSIL/CIN } 3\end{array}$ & 9 & $0(0 \%)$ \\
\hline HSIL/CIN 3-total & 48 & $0(0 \%)$ \\
\hline HSIL/CIN 3 alone & 25 & $0(0 \%)$ \\
\hline $\begin{array}{l}\text { HSIL/CIN } 3 \text { with } \\
\text { LSIL/CIN } 1\end{array}$ & 2 & $0(0 \%)$ \\
\hline $\begin{array}{l}\text { HSIL/CIN } 3 \text { with } \\
\text { LSIL/CIN } 1 \text { and } \\
\text { HSIL/CIN } 2\end{array}$ & 9 & $0(0 \%)$ \\
\hline $\begin{array}{l}\text { HSIL/CIN } 3 \text { with } \\
\text { HSIL/CIN } 2\end{array}$ & 13 & $0(0 \%)$ \\
\hline
\end{tabular}

${ }^{\mathrm{a}}$ The number of positive cases is reported for the first lesion grade in each category.

multiple lesions/grades, the adjacent lower-grade components (LSIL/CIN 1 and HSIL/CIN 2) of these cases also lacked expression. The absence of capsid proteins expression in LSIL-associated HSIL/CIN 3 may be related to molecular alterations that are associated with lesion progression but occur before the morphologic changes take place (eg, integration, gene methylation, etc). However, the exact mechanism responsible for the loss of L1 and L2 expression in these lesions is not known. It is also possible that these different grades of SIL represent independent lesions because of multiple HPV types that are not reactive with the antibodies used in this study. However, the number of specimens containing adjacent lesions of different grades is too small for drawing meaningful conclusions. Nonetheless, the hypothesis suggested by this observation, namely, that the presence of L1 and L2 expression might be used as a marker of absence of adjacent HSIL/CIN 3 in the same specimen, would be extremely useful clinically and warrants further examination.

\section{Acknowledgements}

This study was supported by NIH/NCI/ R21CA150033. Dr Yemelyanova is supported by SPORE in Cervical
Cancer Career Development Award NIH/NCI/ P50 CA098252.

\section{Disclosure/conflict of interest}

The authors declare no conflict of interest.

\section{References}

1 Castle PE, Schiffman M, Wheeler CM, et al. Evidence for frequent regression of cervical intraepithelial neoplasia-grade 2. Obstet Gynecol 2009;113:18-25.

2 Ho GY, Bierman R, Beardsley L, et al. Natural history of cervicovaginal papillomavirus infection in young women. N Engl J Med 1998;338:423-428.

3 Moscicki AB, Shiboski S, Hills NK, et al. Regression of low-grade squamous intra-epithelial lesions in young women. Lancet 2004;364:1678-1683.

4 Doorbar J, Cubie H. Molecular basis for advances in cervical screening. Mol Diagn 2005;9:129-142.

5 Middleton K, Peh W, Southern S, et al. Organization of human papillomavirus productive cycle during neoplastic progression provides a basis for selection of diagnostic markers. J Virol 2003;77:10186-10201.

6 Doorbar J. Papillomavirus life cycle organization and biomarker selection. Dis Markers 2007;23:297-313.

7 Buck CB, Cheng N, Thompson CD, et al. Arrangement of L2 within the papillomavirus capsid. J Virol 2008; 82:5190-5197.

8 Lee H, Lee KJ, Jung CK, et al. Expression of HPV L1 capsid protein in cervical specimens with HPV infection. Diagn Cytopathol 2008;36:864-867.

9 Melsheimer P, Kaul S, Dobeck S, et al. Immunocytochemical detection of HPV high-risk type L1 capsid proteins in LSIL and HSIL as compared with detection of HPV L1 DNA. Acta Cytol 2003;47:124-128.

10 Lin Z, Yemelyanova AV, Gambhira R, et al. Expression pattern and subcellular localization of human papillomavirus minor capsid protein L2. Am J Pathol 2009;174:136-143.

11 Gravitt PE, Peyton C, Wheeler C, et al. Reproducibility of HPV 16 and HPV 18 viral load quantitation using TaqMan real-time PCR assays. J Virol Methods 2003; 112:23-33.

12 Yuan CC, Miley W, Waters D. A quantification of human cells using an ERV-3 real time PCR assay. J Virol Methods 2001;91:109-117.

13 Gambhira R, Karanam B, Jagu S, et al. A protective and broadly cross-neutralizing epitope of human papillomavirus L2. J Virol 2007;81:13927-13931.

14 Firzlaff JM, Kiviat NB, Beckmann AM, et al. Detection of human papillomavirus capsid antigens in various squamous epithelial lesions using antibodies directed against the L1 and L2 open reading frames. Virology 1988;164:467-477.

15 Kurman RJ, Shah KH, Lancaster WD, et al. Immunoperoxidase localization of papillomavirus antigens in cervical dysplasia and vulvar condylomas. Am J Obstet Gynecol 1981;140:931-935.

16 Kurman RJ, Jenson AB, Lancaster WD. Papillomavirus infection of the cervix. II. Relationship to intraepithelial neoplasia based on the presence of specific viral structural proteins. Am J Surg Pathol 1983;7:39-52.

17 Griesser H, Sander H, Hilfrich R, et al. Correlation of immunochemical detection of HPV L1 capsid protein 
in pap smears with regression of high-risk HPV positive mild/moderate dysplasia. Anal Quant Cytol Histol 2004;26:241-245.

18 Griesser H, Sander H, Walczak C, et al. HPV vaccine protein L1 predicts disease outcome of high-risk HPV + early squamous dysplastic lesions. Am J Clin Pathol 2009;132:840-845.

19 Xiao W, Bian M, Ma L, et al. Immunochemical analysis of human papillomavirus L1 capsid protein in liquidbased cytology samples from cervical lesions. Acta Cytol 2010;54:661-667.

$20 \mathrm{Yu} \mathrm{L}$, Wang L, Zhong J, et al. Diagnostic value of p16INK4A, Ki-67, and human papillomavirus L1 capsid protein immunochemical staining on cell blocks from residual liquid-based gynecologic cytology specimens. Cancer Cytopathol 2010;118:47-55.

21 Rauber D, Mehlhorn G, Fasching PA, et al. Prognostic significance of the detection of human papilloma virus L1 protein in smears of mild to moderate cervical intraepithelial lesions. Eur J Obstet Gynecol Reprod Biol 2008;140:258-262.

22 Choi YS, Kang WD, Kim SM, et al. Human papillomavirus L1 capsid protein and human papillomavirus type 16 as prognostic markers in cervical intraepithelial neoplasia 1. Int J Gynecol Cancer 2010;20:288-293.

23 Hilfrich R, Hariri J. Prognostic relevance of human papillomavirus L1 capsid protein detection within mild and moderate dysplastic lesions of the cervix uteri in combination with p16 biomarker. Anal Quant Cytol Histol 2008;30:78-82.

24 Hoshikawa S, Sano T, Yoshida T, et al. Immunohistological analysis of HPV L1 capsid protein and p16 protein in low-grade dysplastic lesions of the uterine cervix. Pathol Res Pract 2010;206:816-820.
25 Negri G, Bellisano G, Zannoni GF, et al. p16 ink4a and HPV L1 immunohistochemistry is helpful for estimating the behavior of low-grade dysplastic lesions of the cervix uteri. Am J Surg Pathol 2008;32:1715-1720.

26 Huang MZ, Li HB, Nie XM, et al. An analysis on the combination expression of HPV L1 capsid protein and p16INK4a in cervical lesions. Diagn Cytopathol 2010; 38:573-578.

27 Yoshida T, Sano T, Kanuma T, et al. Immunochemical analysis of HPV L1 capsid protein and p16 protein in liquid-based cytology samples from uterine cervical lesions. Cancer 2008;114:83-88.

28 Trimble CL, Piantadosi S, Gravitt P, et al. Spontaneous regression of high-grade cervical dysplasia: effects of human papillomavirus type and HLA phenotype. Clin Cancer Res 2005;11:4717-4723.

29 Auvinen E, Kujari H, Arstila P, et al. Expression of the L2 and E7 genes of the human papillomavirus type 16 in female genital dysplasias. Am J Pathol 1992;141: 1217-1224.

30 Galgano MT, Castle PE, Atkins KA, et al. Using biomarkers as objective standards in the diagnosis of cervical biopsies. Am J Surg Pathol 2010;34:1077-1087.

31 Brandsma JL, Sun Y, Lizardi PM, et al. Distinct human papillomavirus type 16 methylomes in cervical cells at different stages of premalignancy. Virology 2009;389: 100-107.

32 Sun C, Reimers LL, Burk RD. Methylation of HPV16 genome $\mathrm{CpG}$ sites is associated with cervix precancer and cancer. Gynecol Oncol 2011;121:59-63.

33 Turan T, Kalantari M, Calleja-Macias IE, et al. Methylation of the human papillomavirus-18 L1 gene: a biomarker of neoplastic progression? Virology 2006; 349:175-183. 